\title{
Energy Transfer in Triple Semiconductor-Organic Hybrid Structures
}

\author{
O. A. Dubovskiy, V. M. Agranovich \\ Institute of Spectroscopy, Russian Academy of Science, Moscow, Russia \\ Email: dubovskiyoa@mail.ru
}

How to cite this paper: Dubovskiy, O.A. and Agranovich, V.M. (2017) Energy Transfer in Triple Semiconductor-Organic Hybrid Structures. Soft Nanoscience Letters, 7, 1-15. https://doi.org/10.4236/snl.2017.71001

Received: August 12, 2017

Accepted: October 17, 2017

Published: October 20, 2017

Copyright $\odot 2017$ by authors and Scientific Research Publishing Inc. This work is licensed under the Creative Commons Attribution International License (CC BY 4.0).

http://creativecommons.org/licenses/by/4.0/

\begin{abstract}
With aim to increase set of modern commercial optoelectronic devices we investigate the optical properties of new triple semiconductor-organics-semiconductor nanostructure having two semiconductor layers with organic layer between. This will be development to majority of modern publications with investigations of only double hybrid nanostructures with one contacting semiconductor layer and one organic layer. It is supposed that the energy of exciton in the first layer is larger than the energy of exciton in organic layer and that the energy of exciton in organic layer is larger in comparison with energy of exciton in second semiconductor layer. It was shown that installation of organics leads to some frequencies at different parameters or to reflection increasing and transmission decrease or to reverted dependence. New recurrent method of inverted calculation for fields is proposed and using this method the frequency dependences of optical characteristics have been calculated. The role of second semiconductor layer in considered triple structure has been estimated.
\end{abstract}

\section{Keywords}

Excitons, Hybrid, Organics, Semiconductor, Nanostructures, Pumping

\section{Introduction}

The majority of modern commercial optoelectronic devices such as LEDs, solar cells, and nonlinear-optical devices are built mainly on the basis of traditional inorganic semiconductors. Over the last couple of decades, however, a lot of progress has been made in producing devices based on organic electronic materials, which, for many applications, may become less expensive alternatives to inorganic counterparts. The development prospects of organic materials are however mostly limited in their scope to relatively low-performance areas. For this 
reason a qualitatively different way of using organic electronic compounds can be via exploiting resonant interactions in organic-inorganic hybrid two layer structures with the organic layer and very thin inorganic layer possessing nearly equal energies of neutral excitations (excitons) [1]. Such approach was widely investigated and the results can be found in many review papers [2]-[11]. Nonradiative energy transfer in a hybrid structure with organic overlayer has been experimentally studied in [2]. The hybrid nanostructure with the InGaN quantum wells as a donor and a monolayer of quantum dots as an acceptor was first studied in [3]. We also mention here investigating of structure, where the temperature dependence of exciton energy transfer was studied [4]. It was shown that organic molecules can be overgrown with $\mathrm{ZnO}$ employing epitaxial methods without degradation of their electronic structure [5]. It was investigated that Forster resonant coupling between the Wannier-Mott and Frenkel excitons in hybrid structures comprising single (Ga, In) N quantum wells spaced from FBDP polymer layers [6]. Details of optical characteristics of hybrid films were investigated in [7]. The results of first experimental observation of Dexter type energy transfer in organic/inorganic hybrid materials were published in [8] [9]. However, in mentioned publications only the nanostructures with one contacting semiconductor thin layer and one organic layer were used.

In this paper we propose to investigate the possibilities which can be created by triple hybrid nanostructure. We investigate the optical properties of more complex triple semiconductor-organics-semiconductor nanostructure with two very thin semiconductor layers and organic layer between them. The thickness of each semiconductor layer must be small as in double semiconductor-organic structure, i.e., approximately $2-4 \mathrm{~nm}$. In contrast to previously published papers we take into account only external pumping wave assuming that thickness of organic layer is larger in comparison with exciton diffusion length in organics. We suppose that in triple structure the energy of Wannier-Mott exciton in the first semiconductor layer is bigger than energy of Frenkel exciton in organic layer as it was assumed usually for double hybrid resonance structures [12] [13]. We assume also that external orthogonal to surfaces optical irradiation produces in the first semiconductor layer the Wannier-Mott excitons with the rather high energy. These excitons can create Frenkel excitons in the intermediate organic region of triple structure and we assume that their energy will be not less than the energy of the Wannier-Mott excitons in the last and lowest third layer of hybrid structure. The role of organics in this structure may be essential since its presence will increase energy flow to second semiconductor layer. The influence of organics may be interesting also in the case when the energies of used semiconductors are resonating with the energies of virtual modes of organic slab [14] [15]. Main equations, new recurrent method of their solution and analytical frequency dependences of reflection and transmission coefficients are presented in Part 2. The influence of organic layer on the frequency of reflection and transmission is discussed and the comparison of spectra without and with in- 
stalled organics defines region of frequencies where organic layer gives positive effect.

\section{Main Equations and Recurrent Procedure of Their Solution for Triple Hybrid}

We investigate triple hybrid structure using set of planes orthogonal to z-direction with electric fields polarized in $\mathrm{x}$-direction and magnetic fields polarized in $\mathrm{y}$-direction. Structure of triple hybrid semiconductor-organic-semiconductor is shown schematically by Figure 1 . This structure includes upper plane of first semiconductor with Wannier-Mott exciton of high energy (WMH). Intermediate organic slab of finite thickness $L$ with Frenkel exciton (FR) is disposed below at some distance $d_{1}$ from WMH. Slab of FR is disposed in interval $0>z>-L$ and slab of WMH is disposed at $z=-L-d_{1}$. Plane of second semiconductor with Wannier-Mott exciton of low energy (WML) is disposed at $z=d_{2}$ below the border $z=0$ of FR. We assume that Wannier-Mott excitons are generated in layer WMH by orthogonal to plane external optical radiation with frequency $\omega$, amplitude of electric strength $E_{1}$ and wave vector along z direction. Reflected from triple hybrid radiation with amplitude $E_{2}$ is shown in the region $z<-L-d_{1}$ by arrow directed up. Arrows in the region $(-L)>z>-L-d_{1}$ between WMH and FR show waves $E_{3}$ and $E_{4}$, running in positive and negative directions of $\mathrm{z}$ axis. Arrows in stroked interval

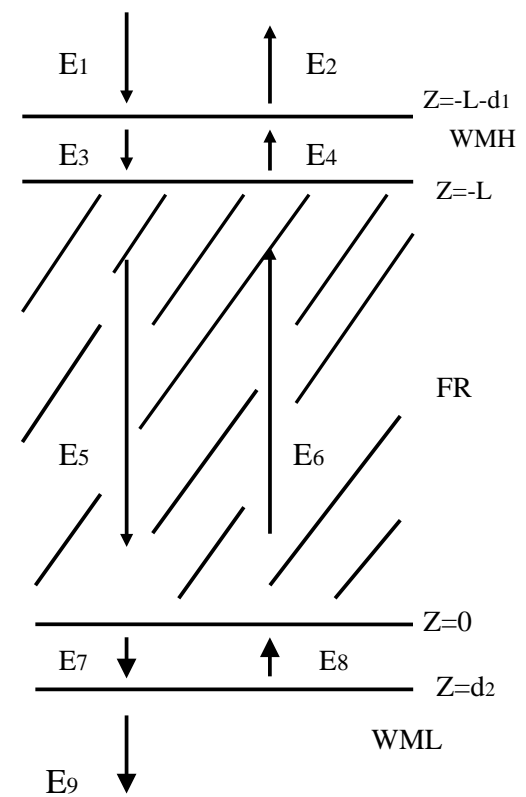

Figure 1. Scheme of triple hybrid structure. The semiconductor with $\mathrm{WMH}$ is shown as upper plane. The semiconductor with WML is shown as lower plane. The organics with FR is shown as intermediate stroked slab between these planes. The arrows demonstrate set of electromagnetic fields including pumping field. The positions of semiconductor planes and borders of organic slab are shown on right side. 
$0>\mathrm{Z}>-L$ of FR indicate waves $E_{5}$ and $E_{6}$ running in organics in positive and negative directions of $z$ axis. We assume that Frenkel excitons are polarized also in $\mathrm{x}$-direction. Arrows in interval $d_{2}>z>0$ indicate waves $E_{7}$ and $E_{8}$ running between FR and WML in positive and negative directions of $z$ axis. Finally arrow in space $z>d_{2}$ indicates transmitting wave $E_{9}$.

We consider the set of linear equations for fields $E_{i}, i=2,3, \cdots, 9$ corresponding to Figure 1 with external field $E_{1}$. This set includes eight equations of continuity for electric and magnetic fields on two planes $\mathrm{WMH}, \mathrm{WML}$ and two borders of FR. We will use below equations for electric and magnetic fields with appropriated frequency dependences of susceptibility for WMH, WML and permeability function of FR. The following well known relation between electric and magnetic fields must be used

$$
H_{y}=(i c / \omega)\left(\partial E_{x} / \partial z\right)
$$

Usual procedure of solution with appropriated determinants for this system of linear equations for $E_{i}, i=2,3, \cdots, 9$ with fixed $E_{1}$ is well known. But for hybrid structures with increasing number of planes rank of determinants drastically increases and using of standard procedure is embarrassing.

We use below inverted procedure considering as known last transmitting wave. In this case for Figure 1 it is the field $E_{9}$. Then simple recurrent procedure without any determinants of high rank defines in inverted order all amplitudes $E_{i}, i=8,7, \cdots, 2$ of all pairs for planes and borders. Start begins from fields $E_{7}, E_{8}$ near shady WML in inverted order and all functions $E_{i}, i=8,7, \cdots, 2$ are defined as functions of field $E_{9}$. Linear dependence of really fixed external field $E_{1}$ is defined on the final stage as function of field $E_{9}$. Inversion of this function defines coefficient of transmission $\left|E_{9} / E_{1}\right|^{2}$. Inversion of all other dependences defines all fields including field $E_{2}$ of reflected wave and coefficient of reflection $\left|E_{2} / E_{1}\right|^{2}$.

Advantage of this reverted procedure with start from WML side is stipulated by possibility to define pair of amplitudes over plane or border as linear functions of known functions under plane or border. Analogous procedure is impossible for start in equations from pumping side because for first plane WMH from two equations must be defined three unknown fields $E_{2}, E_{3}, E_{4}$ and the same in following steps. Only the last step of defining pair of fields under plane as functions of two fields over plane in this direct approach closes procedure by solution of two equations only for $E_{2}$ and $E_{9}$. On the contrary proposed procedure allows simplify analytical calculations for triple structure. Found recurrent relations allow investigating more complex hybrid structures-super lattices with big amount of planes and organic slabs with any order of physical parameters. Calculations below are performed in the same system of non dimensional units that were used in [12] [13] - frequency and oscillators strengths in units of main frequency WMH and length in organic slab thickness L. We subsequently define frequency dependences of successive introduced functions $\Psi_{i, j}(\omega), i<j, i, j=1,2, \cdots, 9$, that defines linear dependences of fields $E_{i}$ on fields 
$E_{j}$ of higher index

$$
E_{i}=\Psi_{i, j}(\omega) E_{j}, i<j .
$$

Beginning with start from shady side two equations of continuity of electric and magnetic fields in plane WML at $z=d_{2}$ have the following forms

$$
\begin{gathered}
E_{7} \mathrm{e}^{i \omega d_{2}}+E_{8} \mathrm{e}^{-i \omega d_{2}}=E_{9} \mathrm{e}^{i \omega d_{2}} \\
E_{7} \mathrm{e}^{\mathrm{i} \omega d_{2}}-E_{8} \mathrm{e}^{-i \omega d_{2}}=E_{9} \mathrm{e}^{i \omega d_{2}}\left(1+i \chi_{2}\right)
\end{gathered}
$$

Susceptibility $\chi_{2}$ of WML in (3b) has the frequency dependence

$$
\chi_{2}(\omega)=\frac{2 \Gamma_{2}}{\omega_{2}-\omega}
$$

Susceptibility (4) includes own frequency $\omega_{2}$ and oscillator strength $\Gamma_{2}$. We have used relation (1) and assume that own frequencies of all triple components are close enough. Solutions of equation system (3) that defines dependences $E_{7}$ and $E_{8}$ from $E_{9}$ have the following forms

$$
E_{7}=\Psi_{7,9} E_{9}, \quad \Psi_{7,9}=\left(1+\frac{i}{2} \chi_{2}\right), \quad E_{8}=\Psi_{8,9} E_{9}, \quad \Psi_{8,9}=\left(\frac{-i}{2} \chi_{2}\right) \mathrm{e}^{2 i \omega d_{2}}
$$

Corresponding continuity equations for electric and magnetic fields on lower border of FR slab have the following forms

$$
E_{5}+E_{6}=E_{7}+E_{8}, \sqrt{\varepsilon\left(E_{5}-E_{6}\right)}=E_{7}-E_{8} .
$$

Frequency dependence of dielectric permeability $\varepsilon(\omega)$ for FR has the standard form

$$
\varepsilon(\omega)=\varepsilon_{0}+\frac{\Gamma_{0}}{\omega_{0}-\omega-i \gamma},
$$

where $\omega_{0}$ is frequency of exciton transition in FR, $\varepsilon_{0}$-constant part of dielectric permeability $\varepsilon(\omega), \Gamma_{0}$ is strength of oscillator for this transition and $\gamma$ defines intrinsic absorption of energy in FR, due to, for example, exciton-phonon interaction.

Solutions of equations system (6) for $E_{5}$ and $E_{6}$ with $E_{7}$ and $E_{8}$ defined by (5) have the following forms

$$
E_{5}=\Psi_{5,9} E_{9}, \quad E_{6}=\Psi_{6,9} E_{9}
$$

Due to increasing complexity of analytical forms of functions $\Psi_{5,9}, \Psi_{6,9}$ and following functions $\Psi_{i, j}$ their exact dependences on frequency and parameters are presented in Appendix. The exact dependences of functions $\Psi_{5,9}, \Psi_{6,9}$ from functions $\Psi_{7,9}, \Psi_{8,9}$ that are defined by (5) are presented in relations (A1) of Appendix.

Equations of continuity for the fields at upper border of FR have the following forms

$$
\begin{aligned}
& E_{3} \mathrm{e}^{-i \omega L}+E_{4} \mathrm{e}^{i \omega L}=E_{5} \mathrm{e}^{-i \sqrt{\varepsilon} \omega L}+E_{6} \mathrm{e}^{i \sqrt{\varepsilon} \omega L} \\
& E_{3} \mathrm{e}^{-i \omega L}-E_{4} \mathrm{e}^{i \omega L}=\sqrt{\varepsilon} E_{5} \mathrm{e}^{-i \sqrt{\varepsilon} \omega L}-\sqrt{\varepsilon} E_{6} \mathrm{e}^{i \sqrt{\varepsilon} \omega L}
\end{aligned}
$$


Solutions of Equations (9) have the following forms

$$
E_{3}=\Psi_{3,5} E_{5}+\Psi_{3,6} E_{6}, E_{4}=\Psi_{4,5} E_{5}+\Psi_{4,6} E_{6} .
$$

The exact analytical dependences of functions $\Psi_{3,5}, \Psi_{3,6}$ and $\Psi_{4,5}, \Psi_{4,6}$ are shown in relations (A2a) and (A2b) of Appendix. Substitution these functions $\Psi_{3,5}, \Psi_{3,6}$ in (10) defines functions $\Psi_{3,9}, \Psi_{4,9}$ as factors connecting $E_{3}, E_{4}$ and $E_{9}$

$$
\begin{aligned}
& E_{3}=\Psi_{3,9} E_{9}, \Psi_{3,9}=\Psi_{3,5} \Psi_{5,9}+\Psi_{3,6} \Psi_{6,9}, \\
& E_{4}=\Psi_{4,9} E_{9}, \Psi_{4,9}=\Psi_{4,5} \Psi_{5,9}+\Psi_{4,6} \Psi_{6,9},
\end{aligned}
$$

Equations for WMH at the distance $d_{1}$ from upper border of FR have the following form

$$
\begin{gathered}
E_{1} \mathrm{e}^{-i \omega\left(L+d_{1}\right)}+E_{2} \mathrm{e}^{i \omega\left(L+d_{1}\right)}=E_{3} \mathrm{e}^{-i \omega\left(L+d_{1}\right)}+E_{4} \mathrm{e}^{i \omega\left(L+d_{1}\right)}, \\
E_{1} \mathrm{e}^{-i \omega\left(L+d_{1}\right)}-E_{2} \mathrm{e}^{i \omega\left(L+d_{1}\right)}=E_{3} \mathrm{e}^{-i \omega\left(L+d_{1}\right)}\left(1+i \chi_{1}\right)-E_{4} \mathrm{e}^{i \omega\left(L+d_{1}\right)}\left(1-i \chi_{1}\right)
\end{gathered}
$$

Susceptibility $\chi_{1}$ of WMH in (12) has the following frequency dependence

$$
\chi_{1}(\omega)=\frac{2 \Gamma_{1}}{\omega_{1}-\omega}
$$

with own frequency $\omega_{1}$ и oscillator strength $\Gamma_{1}$. The solutions of these equations have the following forms

$$
E_{1}=\Psi_{1,3} E_{3}+\Psi_{1,4} E_{4}, E_{2}=\Psi_{2,3} E_{3}+\Psi_{2,4} E_{4} .
$$

The exact analytical dependences of functions $\Psi_{1,3}, \Psi_{1,4}$ and $\Psi_{2,3}, \Psi_{2,4}$ in (14) are presented by relations (A3a) and (A3b) in Appendix. Substitution functions $\Psi_{3,9}, \Psi_{4,9}$ from (11) to (14) define functions $\Psi_{1,9}, \Psi_{2,9}$ in following relations

$$
\begin{aligned}
& E_{1}=\Psi_{1,9} E_{9}, \Psi_{1,9}=\Psi_{1,3} \Psi_{3,9}+\Psi_{1,4} \Psi_{4,9}, \\
& E_{2}=\Psi_{2,9} E_{9}, \Psi_{2,9}=\Psi_{2,3} \Psi_{3,9}+\Psi_{2,4} \Psi_{4,9} .
\end{aligned}
$$

So used recurrent procedure allows to find in (5), (8), (10), (14) and (15) all functions $\Psi_{i, 9}, i=1,2, \cdots, 8$ that define fields $E_{i}=\Psi_{i, 9} E_{9}$ as linear functions of the field $E_{9}$. Solutions for system of dynamic equations in standard direct approach with pumping field $E_{1}$ have the following forms after inverting

$$
E_{i}=\left(\frac{\Psi_{i, 9}}{\Psi_{1,9}}\right) E_{1}, i=2,3, \cdots, 8, E_{9}=\left(\frac{1}{\Psi_{1,9}}\right) E_{1},
$$

All frequency dependences for fields and flows of energy are defined by relations (16) for any region of hybrid. It is evident that function $\Psi_{1,9}$ reveal determinant for system of eight equations. If the external pumping is absent with $E_{1}=0$, it is follow from (16) dispersion equation $\Psi_{1,9}=0$ for finite field $E_{9}$. All own frequencies of hybrid with interacting components are defined from this equation.

The frequency dependences of reflection coefficient $R(\omega)$ and transmission coefficient $T(\omega)$ have as result the following analytical forms 


$$
\begin{aligned}
& R(\omega)=\left|\frac{E_{2}}{E_{1}}\right|^{2}=\left|\frac{\Psi_{2,9}}{\Psi_{1,9}}\right|^{2}=\left|\frac{\sum_{j_{1} j_{2} j_{3}} \Psi_{2, j_{1}} \Psi_{j_{1}, j_{2}} \Psi_{j_{2}, j_{3}} \Psi_{j_{3}, 9}}{\sum_{j_{1} j_{2} j_{3}} \Psi_{1, j_{1}} \Psi_{j_{1}, j_{2}} \Psi_{j_{2}, j_{3}} \Psi_{j_{3}, 9}}\right|^{2} \\
& T(\omega)=\left|\frac{1}{E_{1}}\right|^{2}=\left|\frac{1}{\Psi_{1,9}}\right|^{2}=\left|\frac{1}{\sum_{j_{1} j_{2} j_{3}} \Psi_{1, j_{1}} \Psi_{j_{1}, j_{2}} \Psi_{j_{2}, j_{3}} \Psi_{j_{3}, 9}}\right|^{2}
\end{aligned}
$$

Index summing in (17) must be restricted by nearest "chain" connections that are shown above. It must be noted that in common form found recurrent relations

$$
\begin{gathered}
E_{2 n+1}=\Psi_{2 n+1,2 n+3} E_{2 n+3}+\Psi_{2 n+1,2 n+4} E_{2 n+4}, \\
E_{2 n+2}=\Psi_{2 n+2,2 n+4} E_{2 n+4}+\Psi_{2 n+2,2 n+3} E_{2 n+3}, n=0,1,2, \cdots
\end{gathered}
$$

may be used for defining optical characteristics of super lattices with arbitrary number of planes and arbitrary order of alternation for their parameters. It is possible to use these successive recurrent relations in computer program for investigation of optical and electric characteristics for perovskite materials with structure including alternating regions of contacting organics and semiconductor that leads to extremely high quantum outlet of photoluminescence (90\%) and quantum outlet LED (8\%) [16].

\section{Frequency Dependences of Reflection and Transmission Coefficients for Separate Components of Triple Hybrid}

Investigation of detailed forms of frequency dependence for reflection coefficient and transmission coefficient was done with numerical parameters used in [12] [13] for semiconductors $\mathrm{ZnO}$ or GaAs and anthracene as organic material with $\Omega \simeq 1.5 \mathrm{eV} / \hbar$ and $\pi / L \simeq 2.4 \mathrm{~cm}^{-1}$. We assume that non dimensional own frequencies WMH, FR and WML are quite near and are decreasing in succession $\omega_{1}=1, \omega_{0}=0.96, \omega_{2}=0.925$. This allows do not take into account unimportant non resonant factors. Organics has oscillator strength exceeding oscillator strength of semiconductors. It was taken into account for used set of parameters $\Gamma_{1}=0.002, \Gamma_{0}=0.02, \Gamma_{2}=0.002$.

We consider first simplest case of one isolated WMH at $\Gamma_{0}=0, \Gamma_{2}=0$ for demonstration distinctive analytical frequency dependences of reflection and transmission coefficients. Two equations of continuity for electric and magnetic field in plane $\mathrm{WMH}$ at $z_{1}=-L-d_{1}$ have the following forms

$$
\begin{aligned}
& E_{1} \mathrm{e}^{\mathrm{i} \omega z_{1}}+E_{2} \mathrm{e}^{-i \omega z_{1}}=E_{3} \mathrm{e}^{i \omega z_{1}} \\
& E_{1} \mathrm{e}^{\mathrm{i} \omega z_{1}}-E_{2} \mathrm{e}^{-i \omega z_{1}}=E_{3} \mathrm{e}^{i \omega z_{1}}\left(1+i \chi_{1}\right)
\end{aligned}
$$

Solutions of Equation (18) defining dependences $E_{1}, E_{2}$ from $E_{3}$ have the following forms

$$
E_{1}=\Psi_{1,3} E_{3}, \quad E_{2}=\Psi_{2,3} E_{3} .
$$

The exact analytical dependences of functions $\Psi_{1,3}, \Psi_{2,3}$ in (19) are shown in relations (A4) of Appendix. 
The frequency dependences for reflection coefficient $R_{1}(\omega)$ and transmission coefficient $T_{1}(\omega)$ are defined as

$$
\begin{aligned}
& R_{1}(\omega)=\left|\frac{E_{2}}{E_{1}}\right|^{2}=\left|\frac{\Psi_{2,3}}{\Psi_{1,3}}\right|^{2}=\frac{\Gamma_{1}^{2}}{\left(\omega_{1}-\omega\right)^{2}+\Gamma_{1}^{2}} \\
& T_{1}(\omega)=\left|\frac{1}{E_{1}}\right|^{2}=\left|\frac{1}{\Psi_{1,3}}\right|^{2}=\frac{\left(\omega_{1}-\omega\right)^{2}}{\left(\omega_{1}-\omega\right)^{2}+\Gamma_{1}^{2}}
\end{aligned}
$$

In correspondence with energy conservation the balance equation $R_{1}(\omega)+T_{1}(\omega)=1$ for (20) is correct. Figure 2(a) demonstrates dependence $R_{1}(\omega)$-Lorenz curve in interval $0<R_{1}(\omega)<1$ with maximum $R_{1}\left(\omega_{1}\right)=1$.

For second simple case when only organics is absent at $\Gamma_{0}=0$ for corresponding fields numeration analogous to Figure 1 two equations for plane $z=d_{2}$ have the following forms

$$
\begin{aligned}
& E_{3} \mathrm{e}^{i \omega d_{2}}+E_{4} \mathrm{e}^{-i \omega d_{2}}=E_{5} \mathrm{e}^{\mathrm{i} \omega d_{2}} \\
& E_{3} \mathrm{e}^{\mathrm{i} \omega d_{2}}-E_{4} \mathrm{e}^{-i \omega d_{2}}=E_{5} \mathrm{e}^{\mathrm{i} \omega d_{2}}\left(1+i \chi_{2}\right)
\end{aligned}
$$

Solutions of (21) defining dependences $E_{3}$ and $E_{4}$ from $E_{5}$ have the following forms

$$
E_{3}=\Psi_{3,5} E_{5}, \quad E_{4}=\Psi_{4,5} E_{5} .
$$

The exact analytical dependences of functions $\Psi_{3,5}, \Psi_{4,5}$ in (22) are shown in relations (A5) of Appendix. Equations for WMH have the following forms

$$
\begin{aligned}
& E_{1} \mathrm{e}^{-i \omega\left(L+d_{1}\right)}+E_{2} \mathrm{e}^{i \omega\left(L+d_{1}\right)}=E_{3} \mathrm{e}^{-i \omega\left(L+d_{1}\right)}+E_{4} \mathrm{e}^{i \omega\left(L+d_{1}\right)} \\
& E_{1} \mathrm{e}^{-i \omega\left(L+d_{1}\right)}-E_{2} \mathrm{e}^{i \omega\left(L+d_{1}\right)}=E_{3} \mathrm{e}^{-i \omega\left(L+d_{1}\right)}\left(1+i \chi_{1}\right)-E_{4} \mathrm{e}^{i \omega\left(L+d_{1}\right)}\left(1-i \chi_{1}\right)
\end{aligned}
$$

Solutions of (23) have the following forms

$$
E_{1}=\Psi_{1,3} E_{3}+\Psi_{1,4} E_{4}, \quad E_{2}=\Psi_{2,3} E_{3}+\Psi_{2,4} E_{4} .
$$

The exact analytical dependences of functions $\Psi_{1,3}, \Psi_{1,4}$ and $\Psi_{2,3}, \Psi_{2,4}$ in (24) are shown in relations (A6) of Appendix.

Substitution dependences $E_{3}, E_{4}$ from $E_{5}$ in (22) give dependences $E_{1}, E_{2}$ from $E_{5}$

$$
\begin{array}{ll}
E_{1}=\Psi_{1,5} E_{5}, & \Psi_{1,5}=\Psi_{1,3} \Psi_{3,5}+\Psi_{1,4} \Psi_{4,5} \\
E_{2}=\Psi_{2,5} E_{5}, & \Psi_{2,5}=\Psi_{2,3} \Psi_{3,5}+\Psi_{2,4} \Psi_{4,5}
\end{array}
$$

Then the coefficients of reflection and refraction are defined by following finale relations

$$
\begin{aligned}
& R_{2}(\omega)=\left|\frac{E_{2}}{E_{1}}\right|^{2}=\left|\frac{\Psi_{2,5}}{\Psi_{1,5}}\right|^{2}=\frac{\left|\Psi_{2,3} \Psi_{3,5}+\Psi_{2,4} \Psi_{4,5}\right|^{2}}{\left|\Psi_{1,3} \Psi_{3,5}+\Psi_{1,4} \Psi_{4,5}\right|^{2}} \\
& T_{2}(\omega)=\left|\frac{1}{E_{1}}\right|^{2}=\left|\frac{1}{\Psi_{1,5}}\right|^{2}=\frac{1}{\left|\Psi_{1,3} \Psi_{3,5}+\Psi_{1,4} \Psi_{4,5}\right|^{2}} .
\end{aligned}
$$

Dependence (26a) is shown on Figure 2(b). It is seen additional to Figure 2(a) resonance at $\omega=\omega_{2}$ and both resonances are of quasi Lorenz type due to interaction. 


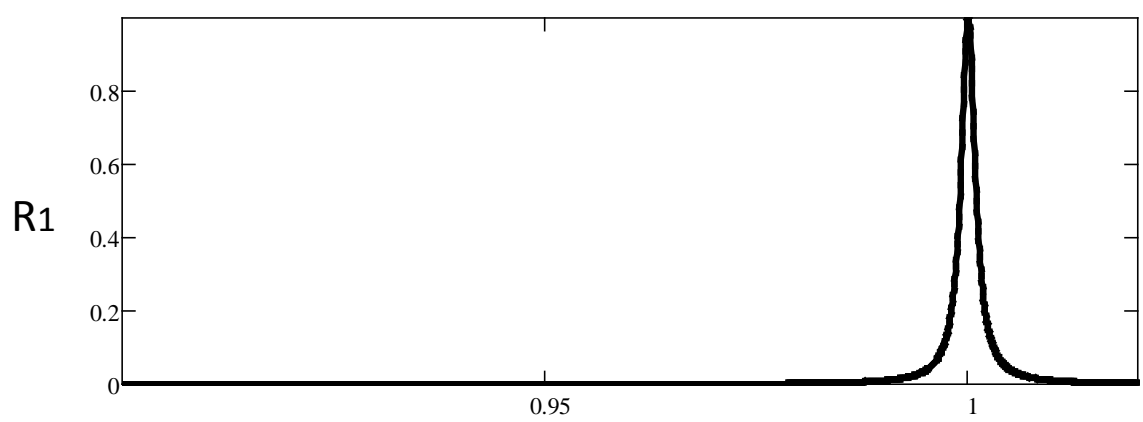

$\omega$

$\omega_{1}$

(a)

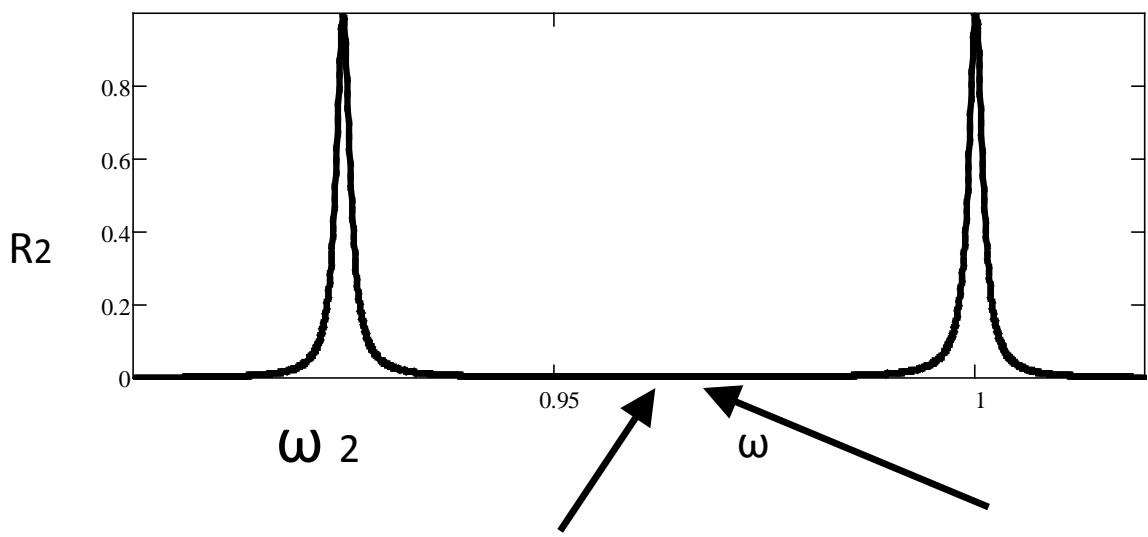

(b)

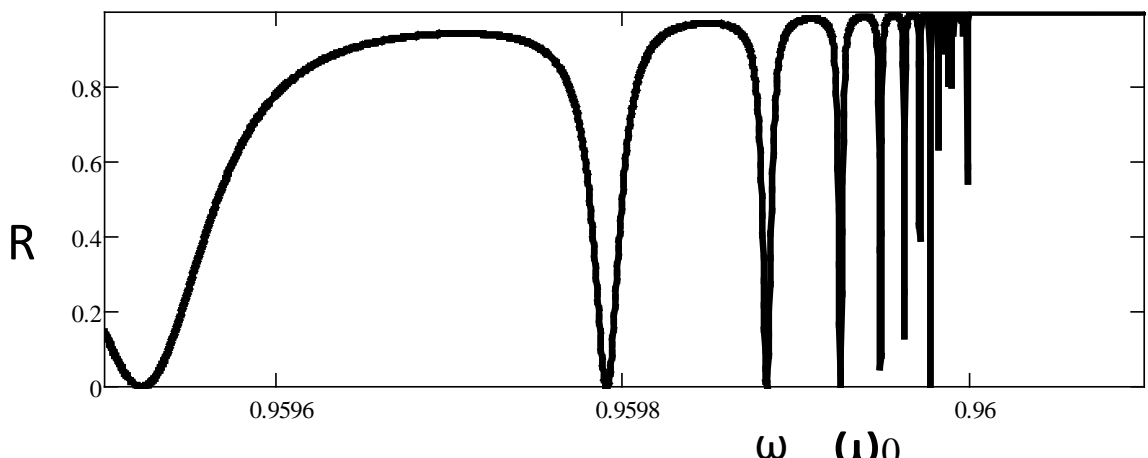

(c)

Figure 2. (a) Reflection spectrum of one isolated semiconductor plane with WMH; (b) Reflection spectrum of two semiconductor planes with WMH and WML without organics; (c) Thin structure of virtual modes in reflection spectrum of isolated organics without semiconductors.

At last it is interesting to consider isolated organics without WMH and WML at $\Gamma_{1}=0, \Gamma_{2}=0$. For this case from (16) at $E_{8}=0$ the following relations define dependences $E_{5}, E_{6}$ from $E_{7}$

$$
\begin{aligned}
& E_{5}=\Psi_{5,7} E_{7}, \Psi_{5,7}=\frac{1}{2}\left(1+\frac{1}{\sqrt{\varepsilon}}\right) \\
& E_{6}=\Psi_{6,7} E_{7}, \Psi_{6,7}=\frac{1}{2}\left(1-\frac{1}{\sqrt{\varepsilon}}\right)
\end{aligned}
$$


Then relations (20) define $E_{3}, E_{4}$ and with (14) reflection coefficient $R(\omega)=\left|E_{4} / E_{3}\right|^{2}$ is defined. Figure 2(c) demonstrates this dependence for $\varepsilon_{0}=1, \gamma=0$. It is seen thin multi resonance structure below $\omega_{0}$ in narrow compared with Figure 2(a), Figure 2(b) interval of frequency. Two arrows from Figure 2(c) to Figure 2(b) show this interval. Detailed calculations with increasing high precision have shown that at frequency approaching to $\omega_{0}$ from left side continuously appears sequences of new resonances. These resonances are condensing below frequency $\omega_{0}$. These additional resonances on Figure 2(c) demonstrate "virtual modes" defined by FR slab thickness, that where investigated in [14] [15]. It was shown in [14] [15] that frequencies of these "virtual modes" are condensing near low frequency side of frequency $\omega_{0}$. These "virtual modes" are presenting the set of points for finite in $\mathrm{z}$ direction $3 \mathrm{D}$ organics that will form dispersion dependence of frequencies for infinite in $\mathrm{z}$ direction crystal. It was found that increasing of intrinsic absorption $\gamma$ leads to decreasing amplitudes and increasing widths of all resonances.

\section{Frequency Dependences of Reflection and Transmission}

The frequency dependences $R(\omega)$ and $T(\omega)$ (17) of fully combined triple hybrid with interacting components are shown on Figure $3(\mathrm{a})$ and Figure $3(\mathrm{~b})$. It was taken into account only one own FR frequency with $\varepsilon_{0}=1$. It is seen that due to relation $R(\omega)+T(\omega)=1$ that is following from Part 2 exactly Figure 3 (b) is mirror copy of Figure 3(a). There are seen on Figure 2(a) two outside quasi Lorenz type curves modified by interaction between components with resonances at frequencies $\omega_{1}$ and $\omega_{2}$ corresponding to WMH and WML. Spectrum of FR on Figure 3(a) is seen in the center between these frequencies. It is seen that at one own frequency of FR below frequency $\omega_{0}$ appears developed set of few resonances.

Figure 4(a) demonstrates difference of reflection coefficients $R d(\omega)$ at installed FR (Figure $3(\mathrm{a}))$ with $R\left(\omega, \Gamma_{0} \neq 0\right)$ and reflection coefficient $R\left(\omega, \Gamma_{0}=0\right)$ (Figure 2(b)) when there is no FR and spectrum of reflection has two Lorenz resonances at $\omega_{1}$ and $\omega_{2}$

$$
R d(\omega)=R\left(\omega, \Gamma_{0} \neq 0\right)-R\left(\omega, \Gamma_{0}=0\right) .
$$

This difference that is up to 10 percent in order has negative sign in region of frequency $\omega_{2}$ and positive sign in other regions including region of frequency $\omega_{1}$. It is seen that near these frequencies function $R d(\omega)$ has oscillations. Fig. $4 \mathrm{~b}$ demonstrates mirror curve for difference of transmission coefficient. Positive sign of $R d(\omega)$ indicates that at frequency $\omega$ of external pumping installation FR increases reflection in outside region of external pumping and decrease energy flow to triple hybrid. Negative sign of $R d(\omega)$ indicates contrary that at frequency $\omega$ of external pumping installation FR decreases reflection in outside region of external pumping and increase energy flow to triple hybrid.

It is important that for frequency $\omega \geq \omega_{1}$ near $\omega_{1}$ at $L=1$ the difference is positive $R d(\omega)>0$ and FR installation increases reflection. It was shown that 


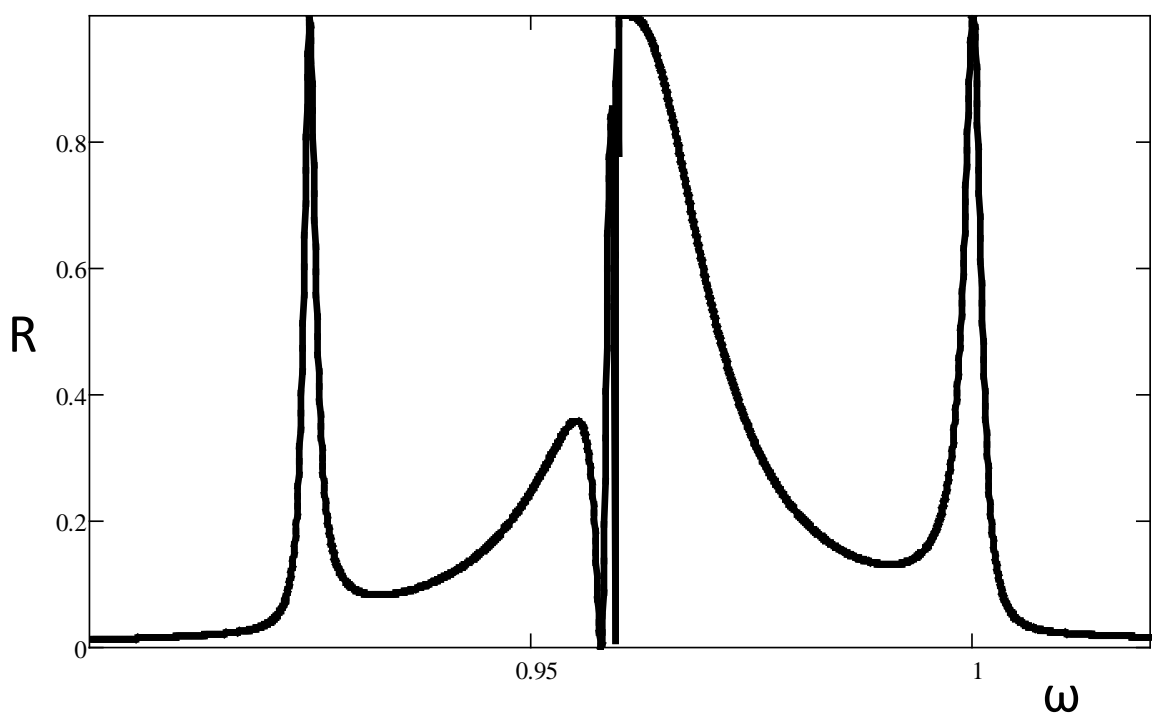

(a)

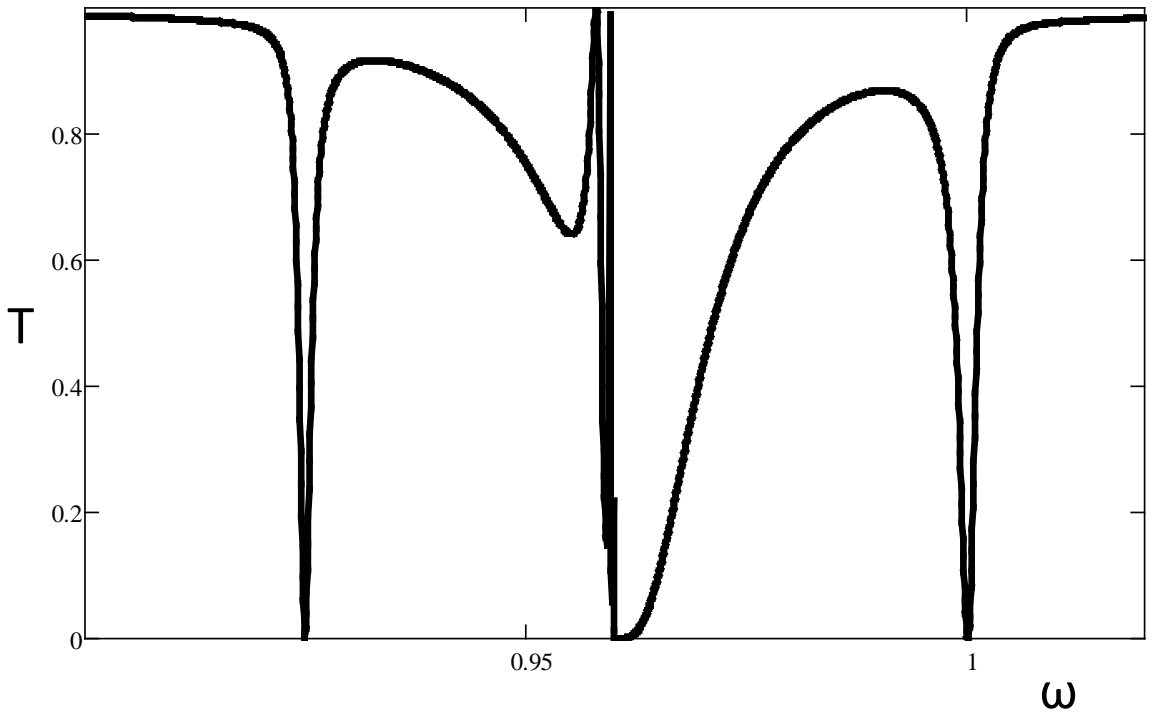

(b)

Figure 3. (a) Frequency dependence of reflection coefficient for triple hybrid structure; (b) Frequency dependence of transmission coefficient for triple hybrid structure.

for $L=0.87$ the function $R d(\omega)<0$ in the same region of frequencies. It means that at this frequency installation of organics in contrary decreases reflection and increases transmission.

We have investigated also spectra of reflection and transmission for different distances $d_{2}$ between FR and WML including case when WML is absent. It was found that WML is working as reflector turning light back to organics that is important for transformation current to light.

\section{Conclusion}

It was shown that installation of organics at external pumping with frequency near own frequency WMH leads to some frequencies at different parameters or 


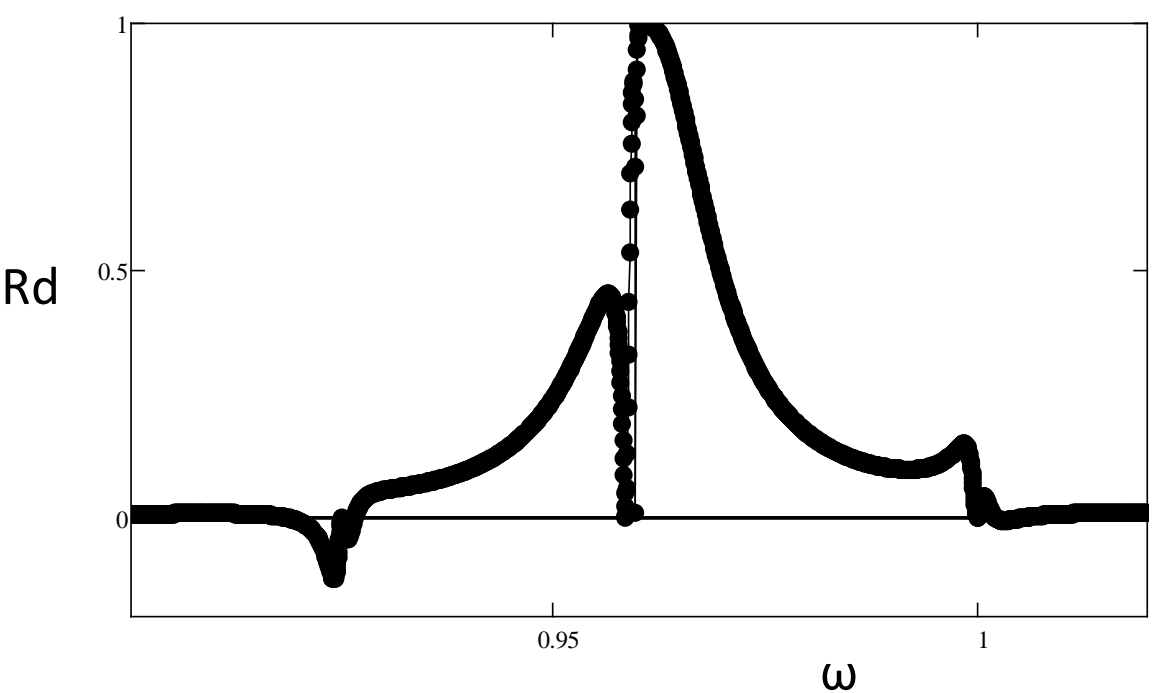

(a)

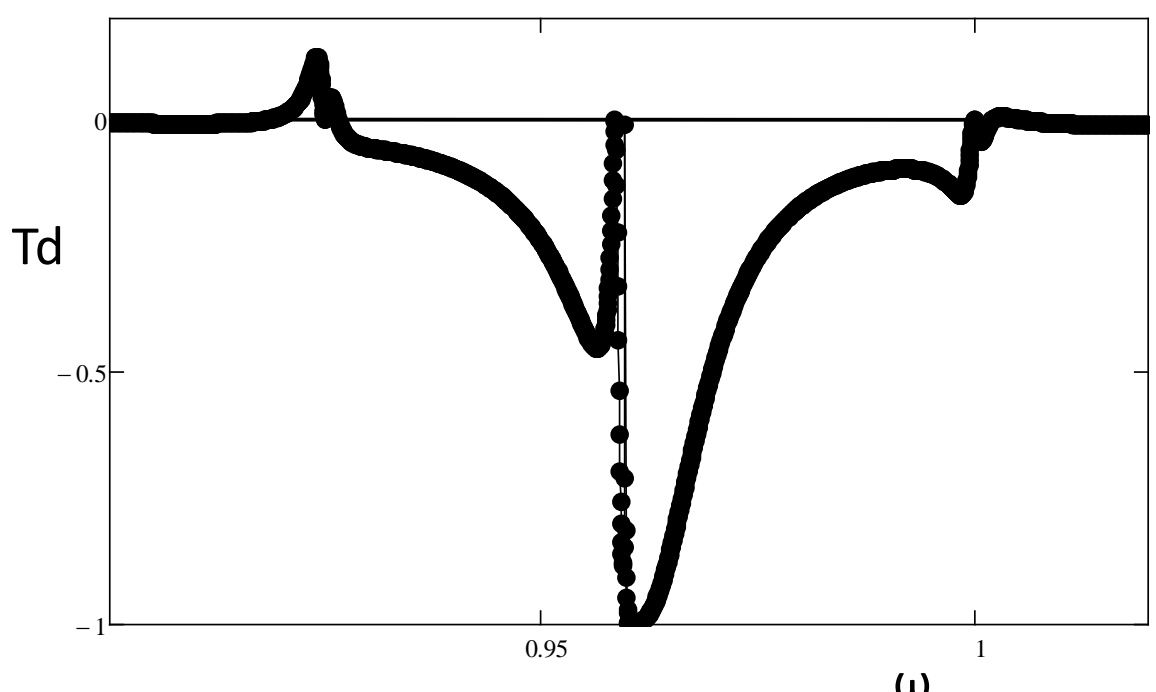

(b)

Figure 4. (a) Difference of reflection coefficients of triple hybrid structure with organics and without organics; (b) Difference of transmission coefficients of triple hybrid structure with organics and without organics.

to reflection increasing and transmission decrease and vice versa to reflection decreasing and transmission increase. The results of this article allow defining regions of parameters and frequency where technological effectiveness may be increased. The proposed new inverted method of calculation allows quick effective defining of optical characteristics for investigated triple hybrid and for super lattices of arbitrary sequence of planes and their parameters. It was found also by comparison spectra of triple hybrid with and without WML that works as reflector turning light back to organics. The proposed new inverted method of calculation may be useful also for reverse technology of transformation light to current by super lattices with arbitrary sequence of planes and their parameters. 


\section{References}

[1] Agranovich, V.M., Gartstein, Yu.N. and Litinskaya, M. (2011) Hybrid Resonant Organic-Inorganic Nanostructures for Optoelectronic Application. Chemical Reviews, 111, 5179-5214. https://doi.org/10.1021/cr100156x

[2] Blumenstegel, S., Sadofev, S, Xu, S.C., Puls, J., and Henneberger, F. (2006) Converting Wannier into Frenkel Exciton in an Inorganic-Organic Hybrid Semiconductor Nanostructure. Physical Review Letters, 97, 247401.

[3] Acherman, M., Petruska, M.A., Kos, S., Smith, D.I., Koleske, D.D. and Klimov, V.I. (2004) Energy-Transfer Pumping of Semiconductor Nanocrystals Using an Epitaxial Quantum Well. Nature, 429, 642. https://doi.org/10.1038/nature02571

[4] Rohmoser, S., Baldauf, J., Harley, R.T., Lagoudakis, P.J., Sapra, S., Euchmuller, A. and Watson, I.M. (2007) Temperature Dependence of Exciton Transfer in Hybrid Quantum Well/Nanocrystal Heterostructures. Applied Physical Letters, 91, Article ID: 92126.

[5] Blumenstegel, S, Sadofev, S., Puls, J. and Henneberger, F. (2009) An Inorganic/Organic Semiconductor "Sandwich" Structure Grown by Molecular Beam Epitaxy. Advanced Matterials, 21, 4850. https://doi.org/10.1002/adma.200900703

[6] Itskos, G., Heliotis, G., Lagoudakis, P.G., Lupton, J., Barradas, N.P., Alves, E., Pereira, S., Watson, I.M., Dawson, M.D., Feldman, J., Murray, R. and Bradley, D.D.C. (2007) Efficient Dipole-Dipole Coupling of Mott-Wannier and Frenkel Excitons in (Ga, In) N Quantum Well/Polyfluorene Semiconductor Heterostructures. Physical Review B, 76, Article ID: 035344. https://doi.org/10.1103/PhysRevB.76.035344

[7] Zhang, Q., Atay, T., Tischler, J.R., Bradley, M.S., Bulovic, V. and Nurmikko, A.V. (2007) Highly Efficient Resonant Coupling of Optical Excitations in Hybrid Organic/Inorganic Semiconductor Nanostructures. Nature Nanotechnology, 2, 555. https://doi.org/10.1038/nnano.2007.253

[8] Ema, K., Inomata, M., Kato, Y., Kunigita, H. and Era, M. (2008) Nearly Perfect Triplet-Triplet Energy Transfer from Wannier Excitons to Naphthalene in Organic-Inorganic Hybrid Quantum-Well Materials. Physical Review Letters, 100, Article ID: 257401. https://doi.org/10.1103/PhysRevLett.100.257401

[9] Ema, K., Umeda, K., Toda, M., Yajima, C., Arai, Y., Kunugita, H., Wolverson, D. and Davies, J.J. (2006) Huge Exchange Energy and Fine Structure of Excitons in an Organic-Inorganic Quantum Well Material. Physical Review B, 73, Article ID: 241310. https://doi.org/10.1103/PhysRevB.73.241310

[10] Smith, R., Liu, B., Bai, J. and Wand, T. (2013) Hybrid III-Nitride/Organic Semiconductor Nanostructure with High Efficiency Nonradiative Energy Transfer for White Light Emitters. Nano Letters, 13, 3042. https://doi.org/10.1021/nl400597d

[11] Schlesinger, R., Bianchi, F., Blumstenger, S., Christodoulou, C., Hennenberger, F. and Koch, N. (2015) Efficient Light Emission from Inorganic and Organic Semiconductor Hybrid Structures by Energy-Level Tuning. Nature Communications, 6, 6754. https://doi.org/10.1038/ncomms7754

[12] Agranovich, V.M., Benisti, H. and Weisbuch, C. (1997) Organic and Inorganic Quantum Wells in Microcavity: Frenkel-Wannier-Mott Excitons Hybridization and Energy Transformations. Solid State Communications, 102, 631-636.

[13] Dubovskiy, O.A. and Agranovich, V.M. (2017) To the Theory of Hybrid Organic/Semiconductor Nanostructures in Microcavity. Solid State Communications, 251, 66-72.

[14] Kliever, K.L. and Fuchs, R. (1966) Optical Modes of Vibration in an Ionic Crystal 
Slab Including Retardation. I. Non radiation Region. Physical Review, 144, 4955-4503.

[15] Kliever, K.L. and Fuchs, R. (1966) Optical Modes of Vibration in an Ionic Crystal Slab Including Retardation. II. Radiation Region. Physical Review, 150, 573-588. https://doi.org/10.1103/PhysRev.150.573

[16] Veldhuis, S.A., Boix, P.P., Yantaro, N., Li, M., Sum, T.C., Mathews, N. and Mhaisalkar, S.G. (2016) Perovskite Materials for Light-Emitting Diodes and Lasers. Advanced Materials, 28, 6804-6834. https://doi.org/10.1002/adma.201600669 


\section{Appendix}

Frequency Dependences of $\Psi_{i, j}$ Functions.

The exact dependences of functions $\Psi_{5,9}, \Psi_{6,9}$ from functions $\Psi_{7,9}, \Psi_{8,9}$ have in (8) the following forms

$$
\begin{aligned}
& \Psi_{5,9}=\frac{1}{2}\left(1+\frac{1}{\sqrt{\varepsilon}}\right) \Psi_{7,9}+\left(1-\frac{1}{\sqrt{\varepsilon}}\right) \Psi_{8,9}, \\
& \Psi_{6,9}=\frac{1}{2}\left(1-\frac{1}{\sqrt{\varepsilon}}\right) \Psi_{7,9}+\left(1+\frac{1}{\sqrt{\varepsilon}}\right) \Psi_{8,9} .
\end{aligned}
$$

The exact analytical dependences of functions $\Psi_{3,5}, \Psi_{3,6}$ and $\Psi_{4,5}, \Psi_{4,6}$ in (10) have the following forms

$$
\begin{aligned}
& \Psi_{3,5}=\frac{1}{2}(1+\sqrt{\varepsilon}) \mathrm{e}^{-i \omega L(\sqrt{\varepsilon}-1)}, \Psi_{3,6}=\frac{1}{2}(1-\sqrt{\varepsilon}) \mathrm{e}^{i \omega L(\sqrt{\varepsilon}+1)} \\
& \Psi_{4,5}=\frac{1}{2}(1-\sqrt{\varepsilon}) \mathrm{e}^{-i \omega L(\sqrt{\varepsilon}+1)}, \quad \Psi_{4,6}=\frac{1}{2}(1+\sqrt{\varepsilon}) \mathrm{e}^{i \omega L(\sqrt{\varepsilon}-1)} .
\end{aligned}
$$

The exact analytical dependences of functions $\Psi_{1,3}, \Psi_{1,4}$ and $\Psi_{2,3}, \Psi_{2,4}$ in (14) have the following forms

$$
\begin{gathered}
\Psi_{1,3}=\left(1+\frac{i}{2} \chi_{1}\right), \quad \Psi_{1,4}=\frac{i}{2} \chi_{1} \mathrm{e}^{2 i \omega\left(L+d_{1}\right)}, \\
\Psi_{2,3}=-\frac{1}{2} i \chi_{1} \mathrm{e}^{-2 i \omega\left(L+d_{1}\right)}, \Psi_{2,4}=\left(1-\frac{i}{2} \chi_{1}\right) .
\end{gathered}
$$

The exact analytical dependences of functions $\Psi_{1,3}, \Psi_{2,3}$ in (19) have the following forms

$$
\Psi_{1,3}=\left(1+\frac{i}{2} \chi_{1}\right), \quad \Psi_{2,3}=\left(\frac{-i}{2} \chi_{1}\right) \mathrm{e}^{2 i \omega z_{1}} .
$$

The exact analytical dependences of functions $\Psi_{3,5}, \Psi_{4,5}$ in (22) have the following forms

$$
\Psi_{3,5}=\left(1+\frac{i}{2} \chi_{2}\right), \quad \Psi_{4,5}=\left(\frac{-i}{2} \chi_{2}\right) \mathrm{e}^{2 i \omega d_{2}} .
$$

The exact analytical dependences of functions $\Psi_{1,3}, \Psi_{1,4}$ and $\Psi_{2,3}, \Psi_{2,4}$ in (24) have the following forms

$$
\begin{aligned}
& \Psi_{1,3}=\left(1+\frac{i}{2} \chi_{1}\right), \quad \Psi_{1,4}=\frac{i}{2} \chi_{1} \mathrm{e}^{2 i \omega\left(L+d_{1}\right)} \\
& \Psi_{2,3}=-\frac{1}{2} i \chi_{1} \mathrm{e}^{-2 i \omega\left(L+d_{1}\right)}, \Psi_{2,4}=\left(1-\frac{i}{2} \chi_{1}\right)
\end{aligned}
$$

